\title{
Antibiotic Overuse for COVID-19: Are We Adding Insult to Injury?
}

\author{
Seid Getahun Abdela, ${ }^{1 \star}$ Laurens Liesenborghs, ${ }^{9}$ Fentaw Tadese, ${ }^{1}$ Seid Hassen Abegaz, ${ }^{2}$ Fentaw Bialfew Bayuh, ${ }^{2}$ \\ Etsegent Arega Asmamaw, ${ }^{3}$ Tamiru Assefa Mebrate, ${ }^{3}$ Abreham Eshetu Mamo, ${ }^{3}$ Wendemaegn Embiale, ${ }^{4,5}$ Samuel Hunegnaw, ${ }^{4}$ \\ Dereje Bedanie Hundie, ${ }^{4}$ Zewdu Hurissa, ${ }^{6}$ Gebi Agero, ${ }^{6}$ Abdene Weya Kaso, ${ }^{7}$ Maraki Assefa Mebrate, ${ }^{8}$ Saskia van Henten, ${ }^{9}$ and \\ Johan van Griensven ${ }^{9}$ \\ ${ }^{1}$ College of Medicine and Health Sciences, Wollo University, Dessie, Ethiopia; ${ }^{2}$ Boru Meda General Hospital, Dessie, Ethiopia; ${ }^{3}$ Saint Peter \\ Specialized Hospital, Addis Ababa, Ethiopia; ${ }^{4}$ College of Medicine and Health Sciences, Bahir Dar University, Bahir Dar, Ethiopia; ${ }^{5}$ Department \\ of dermatology, Amsterdam Institute for Infection and Immunity (Al and II), Amsterdam UMC, University of Amsterdam, Amsterdam, The \\ Netherlands; ${ }^{6}$ College of Health Sciences, Arsi University, Asella, Ethiopia; ${ }^{7}$ College of Health Sciences, Dilla University, Dilla, Ethiopia; \\ ${ }^{8}$ Ethiopian Airlines Medical Unit Services; ${ }^{9}$ Department of Clinical Sciences, Institute of Tropical Medicine, Antwerp, Belgium
}

\begin{abstract}
In this study, we described the proportion of COVID-19 patients started on antibiotics empirically and the work-ups performed to diagnose bacterial superinfection. We used a retrospective cohort study design involving medical records of symptomatic, hospitalized COVID-19 patients who were admitted to these centers. A total of 481 patients were included, with a median age of 41.0 years (interquartile range, 28-58.5 years). A total of $72.1 \%(N=347)$ of COVID-19 patients received antibiotics, either before or during admission. This is troublesome because none of the patients' bacterial culture or inflammatory markers, such as the erythrocyte sedimentation rate or C-reactive protein, were evaluated, and only 73 (15.2\%) underwent radiological investigations. Therefore, national COVID-19 guidelines should emphasize the rational use of antibiotics for the treatment of COVID-19, a primarily viral disease. Integrating antimicrobial stewardship into the COVID-19 response and expanding microbiological capacities in low-income countries are indispensable. Otherwise, we risk one pandemic aggravating another.
\end{abstract}

Frequent and often inappropriate antibiotic use is common in low- and middle-income countries. ${ }^{1}$ Because of the worldwide increase in antimicrobial resistance, this is concerning. Moreover, we fear that the emergence of COVID-19 could further fuel the antimicrobial resistance pandemic. The role of antibiotics in the treatment of COVID19 is not clearly defined. Many national and international guidelines, including Ethiopia's, recommend the use of broad-spectrum antibiotics for patients with COVID-19 to treat any bacterial superinfection. ${ }^{2}$ Yet, the limited available evidence suggests that a bacterial superinfection is not a prominent feature of COVID-19. ${ }^{3}$ Nevertheless, antibiotic consumption is high among COVID-19 patients in most high-income settings ${ }^{4,5}$; however, it has not been assessed in African settings, where the diagnosis of bacterial coinfection is often difficult because of limited investigation modalities. We studied the antibiotic use by COVID-19 patients admitted to four Ethiopian treatment centers between May and November 2020, and assessed the work-up they underwent to diagnose bacterial superinfection.

The management of COVID-19 at the treatment centers is in line with the national treatment guidelines. Patients with an uncomplicated upper respiratory tract viral infection and nonspecific symptoms such as fever, fatigue, cough, or headache are considered to have a mild infection. Among adults, moderate illness is described as mild pneumonia according to the CURB-65 criteria (absence of confusion, urea, respiratory rate, and blood pressure, and age 65 years or older). Severe illness is described as severe pneumonia, acute respiratory distress syndrome, or sepsis responding to noninvasive management. Critical illness is considered when

*Address correspondence to Seid Getahun Abdela, College of Medicine and Health Sciences, Wollo University Dessie, P.O. Box 828, Melaku 7, Ethiopia. E-mail: Seidgech014@gmail.com patients do not respond to noninvasive management, or when there is respiratory failure, septic shock, and/or multiple organ dysfunction or failure. ${ }^{2}$

A total of 481 patients with a median age of 41.0 years (interquartile range, 28-58.5) were included. The majority of patients were male $(N=342 ; 71.1 \%)$, and $190(39.5 \%)$ had at least one comorbidity. More than half of them had mild cases $(N=242 ; 50.3 \%), 78$ had moderate cases $(16.2 \%)$, and 161 had severe or critical cases (33.5\%) (Table 1). Comorbidities were documented among 190 patients (39.5\%); hypertension $(91 ; 47.9 \%)$ and diabetes mellitus $(67$; $35.3 \%$ ) were the two most frequent comorbidities. Chronic respiratory conditions were reported among 23 patients (12.1\%). The median hospital stay was 14 days (interquartile range, $10-17.5$ days), and 58 patients (12\%) died of COVID19 complications.

A total of $72.1 \%(N=347)$ of COVID-19 patients received antibiotics, either before or during admission. Amoxicillin/ clavulanate and azithromycin were the most used oral antibiotics, whereas ceftriaxone and vancomycin were the most frequently prescribed intravenous drugs. Nineteen patients $(5.6 \%)$ received meropenem. This is troublesome because none of the patients' bacterial culture or inflammatory markers, such as the erythrocyte sedimentation rate or C-reactive protein, were evaluated, and only 73 (15.2\%) underwent radiological investigations.

National COVID-19 guidelines should emphasize the rational use of antibiotics for the treatment of COVID-19, which is a primarily viral disease. Antibiotics, especially broadspectrum agents like meropenem, should be used only after careful consideration, and, ideally, they should be accompanied by bacterial culture and antimicrobial susceptibility tests. Integrating antimicrobial stewardship into the COVID19 response and expanding microbiological capacities in low-income countries are indispensable. Otherwise, we risk one pandemic aggravating another. 
TABLE 1

Antibiotics use before and after admission according to the severity status of COVID-19 patients treated across different treatment centers in Ethiopia in 2020

\begin{tabular}{|c|c|c|c|c|}
\hline & $\begin{array}{c}\text { Mild } \\
N=242\end{array}$ & $\begin{array}{c}\text { Moderate } \\
N=78\end{array}$ & $\begin{array}{l}\text { Severe and critical } \\
\qquad N=161\end{array}$ & $\begin{array}{l}\text { Total } \\
N=481\end{array}$ \\
\hline Received antibiotics before admission & $16(2.5 \%)$ & $25(32.1 \%)$ & $46(28.6 \%)$ & $87(18.1 \%)$ \\
\hline Oral route & $6(37.5 \%)$ & $14(56.0 \%)$ & $11(23.9 \%)$ & $31(35.6 \%)$ \\
\hline Parenteral route & $7(43.8 \%)$ & $6(24.0 \%)$ & $29(63.0 \%)$ & $42(48.3 \%)$ \\
\hline Both & $3(18.8 \%)$ & $5(20.0 \%)$ & $6(13.0 \%)$ & $14(16.1 \%)$ \\
\hline Received antibiotics during admission & $110(45.5 \%)$ & $73(93.6 \%)$ & $159(98.8 \%)$ & $342(71.1 \%)$ \\
\hline Oral route & $81(73.6 \%)$ & $24(32.9 \%)$ & $3(1.9 \%)$ & $108(31.6 \%)$ \\
\hline Parenteral route & $26(23.6 \%)$ & $23(31.5 \%)$ & $108(67.9 \%)$ & $157(45.9 \%)$ \\
\hline Both & $3(2.7 \%)$ & $26(35.6 \%)$ & $48(30.2 \%)$ & $77(22.5 \%)$ \\
\hline \multicolumn{5}{|l|}{ Type of antibiotics } \\
\hline Penicillin & $42(17.4 \%)$ & $30(38.5 \%)$ & $19(11.8 \%)$ & $91(18.9 \%)$ \\
\hline Macrolides & 77 (31.8\%) & $45(57.7 \%)$ & 51 (31.7\%) & $173(36.0 \%)$ \\
\hline Third-generation cephalosporin & $33(13.6 \%)$ & $48(61.5 \%)$ & $135(83.9 \%)$ & $216(44.9 \%)$ \\
\hline Fourth-generation cephalosporin & $0(0 \%)$ & $6(7.7 \%)$ & $26(16.1 \%)$ & $32(6.7 \%)$ \\
\hline Fluoroquinolones & $3(1.2 \%)$ & $5(6.4 \%)$ & $8(5.0 \%)$ & $16(3.3 \%)$ \\
\hline Meropenem & $0(0 \%)$ & $0(0 \%)$ & $20(12.4 \%)$ & $20(4.2 \%)$ \\
\hline Vancomycin & $3(1.2 \%)$ & $22(28.2 \%)$ & $118(73.3 \%)$ & $143(29.7 \%)$ \\
\hline \multicolumn{5}{|l|}{ Investigations performed } \\
\hline X-ray imaging & $6(2.5 \%)$ & $13(16.7 \%)$ & $34(21.1 \%)$ & $53(11.0 \%)$ \\
\hline Chest computed tomography scan & $0(0.0 \%)$ & $5(6.4 \%)$ & $15(9.3 \%)$ & $20(4.2 \%)$ \\
\hline Complete blood count & $34(14.0 \%)$ & $39(50.0 \%)$ & $95(59.0 \%)$ & $168(34.9 \%)$ \\
\hline
\end{tabular}

Received May 27, 2021. Accepted for publication September 19, 2021.

Published online October 29, 2021.

Acknowledgments: We thank the management and staff of treatment centers. The American Society of Tropical Medicine and Hygiene has waived the Open Access fee for this article due to the ongoing COVID-19 pandemic and has assisted with publication expenses.

Authors' addresses: Seid Getahun Abdela, Department of Internal Medicine, Wollo University, Dessie, Ethiopia, E-mail: seidgech014@ gmail.com. Laurens Liesenborghs, Saskia van Henten, and Johan van Griensven, Department of Clinical Sciences, Institute of Tropical Medicine, Antwerp, Belgium, E-mails: Iliesenborghs@itg.be, svanhenten@itg.be, and jvangriensven@itg.be. Fentaw Tadese, Department of Public Health, Wollo University, Dessie, Ethiopia, E-mail: fentadese760@yahoo.com. Seid Hassen Abegaz, Boru Meda Hospital, Desse, Ethiopia, E-mail: seidhassen09@gmail.com. Fentaw Bialfew Bayuh, Department of Public Health, Boru Meda Hospital, Desse, Ethiopia, E-mail: fentawbi@gmail.com. Etsegent Arega Asmamaw, St. Peters Hospital, Clinical Governance and Quality Department, Quality Officer, Addis Ababa, Ethiopia, E-mail: etsegenet2010@gmail.com. Tamiru Assefa Mebrate, chief executive director, St. Peters Hospital, Addis Ababa, Ethiopia, E-mail: atamiru2006@gmail.com. Abreham Eshetu Mamo, Academic and research directorate, Director, Saint Peter Specialized Hospital, Addis Ababa, Ethiopia, E-mail: biniamnesru.1234@gmail.com. Maraki Assefa Mebrate, Ethiopian Airlines Medical Unit Services, Addis Ababa, Ethiopia, E-mail: assefamaraki@gmail.com. Wendemaegn Embiale, Department of Dermatovenerology, Bahir dar University, Bahir Dar, Ethiopia, E-mail: wendaab@gmail.com. Samuel Hunegnaw, Department of Internal Medicine, Bahir Dar University College of Medical and Health Sciences, Bahir Dar, Amhara, Ethiopia, E-mail: hunegnawsamuel@gmail.com. Dereje Bedanie Hundie, Department of Medicine, College of Medicine and Health Sciences, Bahir Dar University, Bahir Dar, Ethiopia, E-mail: derejebedane@gmail.com. Zewdu Hurissa, Department of Internal Medicine, Arsi University College of Health Sciences, Asella, Oromia, Ethiopia, E-mail: hurzew@yahoo.com. Gebi Agero, Department of Public Health, Arsi University College of Health Sciences, Asella, Oromia, Ethiopia, E-mail: gebi.agero@yahoo.com. Abdene Weya Kaso, Department of Public Health, Dilla University College of Health Sciences, Dilla, Ethiopia, E-mail: abdannekaso@gmail.com.

This is an open-access article distributed under the terms of the Creative Commons Attribution (CC-BY) License, which permits unrestricted use, distribution, and reproduction in any medium, provided the original author and source are credited.

\section{REFERENCES}

1. Do NT et al., 2021. Community-based antibiotic access and use in six low-income and middle-income countries: a mixedmethod approach. Lancet Glob Health 9: e610-e619.

2. Federal Ministry of Health Ethiopia, 2020. National Comprehensive COVID19 Management Handbook. Ethiopian Federal Ministry of Health. 2020 Apr.

3. Langford BJ, So M, Raybardhan S, Leung V, Westwood D, MacFadden DR, Soucy JP, Daneman N, 2020. Bacterial co-infection and secondary infection in patients with COVID19: a living rapid review and meta-analysis. Clin Microbiol Infect 26: 1622-1629.

4. Abelenda-Alonso G, Padullés A, Rombauts A, Gudiol C, Pujol M, Alvarez-Pouso C, Jodar R, Carratalà J, 2020. Antibiotic prescription during the COVID-19 pandemic: a biphasic pattern. Infect Control Hosp Epidemiol 41: 1371-1372.

5. Shin DH, Kang M, Song KH, Jung J, Kim ES, Kim HB, 2020. A call for antimicrobial stewardship in patients with COVID-19: a nationwide cohort study in Korea. Clin Microbiol Infect. 27: 653-655. 